\title{
Nivolumab in pre-treated malignant pleural mesothelioma: real-world data from the Dutch expanded access program
}

\author{
Luca Cantini $^{1,2,3 \#}$, Robert A. Belderbos ${ }^{1,2 \#}$, Cornedine J. Gooijer ${ }^{4}$, Daphne W. Dumoulin ${ }^{1,2}$, \\ Robin Cornelissen $^{1,2}$, Sara Baart ${ }^{1,2,5}$, Jacobus A. Burgers ${ }^{4}$, Paul Baas ${ }^{4}$, Joachim G. J. V. Aerts ${ }^{1,2}$ \\ ${ }^{1}$ Department of Pulmonary Medicine, Erasmus MC Rotterdam, Rotterdam, The Netherlands; ${ }^{2}$ Erasmus MC Cancer Institute, Erasmus MC \\ Rotterdam, Rotterdam, The Netherlands; ${ }^{3}$ Clinical Oncology, Marche Polytechnic University, AOU Ospedali Riuniti Ancona, Ancona, Italy; \\ ${ }^{4}$ Department of Thoracic Oncology, Netherlands Cancer Institute, Amsterdam, The Netherlands; ${ }^{5}$ Department of Biostatistics, Erasmus MC, \\ Rotterdam, the Netherlands \\ Contributions: (I) Conception and design: L Cantini, RA Belderbos, CJ Gooijer, R Cornelissen, DW Dumoulin, JA Burgers; (II) Administrative \\ support: All authors; (III) Provision of study materials or patients: All authors; (IV) Collection and assembly of data: L Cantini, RA Belderbos, \\ CJ Gooijer; (V) Data analysis and interpretation: L Cantini, RA Belderbos, S Baart; (VI) Manuscript writing: All authors; (VII) Final approval of \\ manuscript: All authors. \\ \#These authors contributed equally to this work. \\ Correspondence to: Prof. Joachim G. J. V. Aerts. Erasmus MC, Dr. Molewaterplein 40, 3015 GD Rotterdam, The Netherlands. \\ Email: j.aerts@erasmusmc.nl.
}

Background: Randomized phase III trials are ongoing to investigate the efficacy of nivolumab in malignant pleural mesothelioma (MPM), but real-world data are still scarce. In this real-world study, we investigated the clinical outcomes of nivolumab treatment in pre-treated MPM patients.

Methods: Data from 107 nivolumab treated MPM patients within the Dutch expanded access program were retrospectively analyzed. Treatment was independent of programmed death ligand 1 (PD-L1) expression on tumor samples. Univariable and multivariable analyses were performed to evaluate the relationship between clinically important factors, baseline peripheral blood parameters and survival. The landmark method was used to compare the outcome of patients according to their radiological response.

Results: In the full cohort, the median progression-free survival (mPFS) was 2.3 months (95\% CI: 1.6-2.9) and the median overall survival (mOS) was 6.7 months (95\% CI: 6.2-10.0). After 12 weeks, the disease control rate (DCR) was $37 \%$ and the objective response rate (ORR) was 10\%. PD-L1 status was determined in 33 patients $(30 \%)$ and PD-L1 positivity $(\geq 1 \%)$ was associated with an improved ORR (36\% vs. 9\%, P value 0.05 ), but not with PFS or OS. Low albumin was associated with worse OS (P value 0.002). Median OS was significantly longer for patients who had partial response to treatment ( $\mathrm{P}$ value 0.0002).

Conclusions: In this real-world analysis, ORR and mOS were lower compared to those obtained in phase II trials. However, exceptional survival rates were observed in patients who had a radiological response. Although we cannot determine whether prognostic or predictive, PD-L1 expression and albumin were associated with greater response rate and may represent useful biomarkers for nivolumab treatment in MPM.

Keywords: Checkpoint inhibitors; immunotherapy; malignant pleural mesothelioma (MPM); nivolumab; PD-L1

Submitted Dec 20, 2019. Accepted for publication Jun 09, 2020.

doi: $10.21037 /$ tlcr-19-686

View this article at: http://dx.doi.org/10.21037/tlcr-19-686 


\section{Introduction}

Malignant pleural mesothelioma (MPM) is an uncommon but aggressive neoplasm with low survival rates $(1,2)$. Current first-line treatment consists of combination chemotherapy with platinum and anti-folate agents $(1,3)$, with the possible addition of bevacizumab (2). Historically, no therapeutic agent has shown strong activity against mesothelioma in second or third-line treatment (4). The breakthrough of checkpoint inhibitors (CIs) in solid tumors has led to their investigation in MPM patients as well (5). Despite promising results in phase I/II trails with CIs, phase III trials investigating both single agent anticytotoxic T-lymphocyte-associated protein 4 (CTLA4) and anti-programmed cell death 1 (PD-1) treatments failed to show efficacy $(6,7)$. Recently, the PROMISE-meso, a phase III randomized clinical trial (RCTs), comparing the PD1 CI pembrolizumab to chemotherapy (gemcitabine or vinorelbine) as second-line treatment, failed to show superiority of the anti-PD-1 treatment for the primary endpoint progression free survival (PFS) (7). The objective response rate (ORR) was significantly higher in the pembrolizumab arm (22\%) than in the chemotherapy arm $(6 \%)$, but duration of response (DoR) and overall survival (OS) were equal. Nivolumab, another PD-1 inhibitor, showed promising results in phase II trials in pretreated MPM patients (with ORR up to 29\%) (8-11) and is currently being tested in the context of phase III RCTs (NCT03063450, NCT02899299).

Only one study has reported real-world data on second or third-line PD-1 inhibition (pembrolizumab) in MPM (12). In this study, both PFS and OS did not match phase II trial results which could be explained by the use of strict inclusion criteria in the clinical trials $(9,11)$. Outside of clinical trials, there are no reports on the role of nivolumab in pre-treated MPM patients. Most probably, as already observed in phase II/III trials, a small group of MPM patients might benefit from CI treatment.

Relevant biomarkers for response have not yet been determined in this specific setting of MPM. Programmed death-ligand 1 (PD-L1) expression on tumor cells has a controversial role in predicting outcome in MPM $(8,12)$. The low predictive value of PD-L1 expression in MPM has been explained by intra-patient heterogeneity, different cut-off points for PD-L1 positivity and the use of different immunohistochemistry (IHC) markers $(8,12)$. Likewise different cancer types $(13,14)$, other tumor and patient characteristics, as well as peripheral blood values should then be investigated in MPM patients treated with nivolumab, to identify biomarkers for response.

Since February 2018, nivolumab has been provided to MPM patients in the Netherlands through an expanded access program (EAP). This program has offered the unique opportunity to conduct a real-world analysis to investigate the outcome of nivolumab in a population of MPM patients pre-treated with antifolate and platinumbased chemotherapy. Furthermore, we extensively analyzed the correlation between clinically important factors, baseline peripheral blood parameters and clinical outcomes. The impact of radiological response on outcome was also investigated. We present the following article in accordance with the Strengthening the Reporting of Observational Studies in Epidemiology (STROBE) Reporting Checklist (available at http://dx.doi.org/10.21037/tlcr-19-686).

\section{Methods}

\section{Patients}

We retrospectively reviewed data from all 135 MPM patients enrolled at the Erasmus Medical Center (Rotterdam, NL) and The Netherlands Cancer Institute (Amsterdam, NL) in the EAP for nivolumab. Patients had a cytological and/or histological proven MPM and progression after at least one previous line of chemotherapy. Inclusion in the program was independent of PD-L1 expression on tumor samples, which was assessed by IHC using the Ventana SP263 or the Dako 22C3 assays. A recent tumor biopsy was not mandatory. Patients were excluded if they had received any immunotherapy as firstline or maintenance treatment. Patients with a follow-up shorter than 3 months were also excluded from the analysis, unless they progressed or died earlier. Nivolumab was given intravenously at a dose of $3 \mathrm{mg} / \mathrm{kg}$ every 2 weeks. Radiological tumor assessment was carried out 6 weeks $( \pm 1)$ after start of treatment and every 6 weeks $( \pm 1)$ until progression depending on previous computed tomography (CT) evaluation.

\section{Data collection}

Patient and tumor characteristics, as well as radiological response data and blood count parameters within 14 days before the initiation of nivolumab treatment were collected from the digital patient register. The following variables were collected and investigated in statistical analyses: age, 
gender (male $v s$. female), histologic subtype (non-epithelioid vs. epithelioid), Eastern Cooperative Oncology Group (ECOG) Performance Status (PS) at start of nivolumab ( 0 vs. $\geq 1$ ), clinical TNM stage [stage III/IV vs. I/II (VIII edition)] (15), line of treatment (later-lines $v s$. secondline), PD-L1 status (considered as positive if tumor cell expression levels were $\geq 1 \%$, negative if $<1 \%$ ), time to progression (TTP) to previous line of chemotherapy ( $<6 v s . \geq 6$ months), time interval (TI) from diagnosis to start of nivolumab, body mass index (BMI). Albumin values (as continuous variable), platelet count (as continuous variable), and absolute counts for neutrophils, monocytes, eosinophils and lymphocytes were also collected.

Tumor response was assessed using a combination of modified Response Evaluation Criteria In Solid Tumors (mRECIST) for mesothelioma version 1.0 and RECIST modified for immunotherapeutic agents (iRECIST) $(16,17)$. Per iRECIST, if tumor imaging shows initial progression of disease (PD), tumor assessment should be repeated 4 to 8 weeks later in order to confirm PD with the option of continuing treatment if the patient is clinically stable. Patients who had confirmed disease progression by iRECIST discontinued treatment, and the date of the initial CT scan was taken as the time of progression. OS was defined as the time from first CI administration to death from any cause, censored at the last tumor assessment date for patients who were alive at the time of data cutoff. PFS was measured from the time of nivolumab initiation to clinical or radiological progression or death from any cause. ORR was defined as the proportion of patients who had a partial (PR) or complete response (CR) to therapy and DCR as the percentage of patients who achieved complete response, partial response and stable disease (SD). A cutoff of 12 weeks $( \pm 2)$ was selected for both ORR and DCR, according to the majority of RCTs investigating CIs in MPM. DoR was defined as the time from documentation of tumor response to disease progression.

\section{Statistical analysis}

Patient and disease characteristics were reported using count and percentage for categorical variables, median and range for continuous variables. Median PFS and OS were estimated by the Kaplan-Meier method. Differences in probability of surviving between the strata were evaluated by log-rank (Mantel-Cox) test and Bonferroni's correction was used for comparison between more than two groups. The landmark method was used for handling immortal time bias when comparing the outcome of patients according to their radiological response (18). For this specific analysis, all the patients who died before 12 weeks were excluded. A landmark of 12 weeks was chosen because at that time ORR was also calculated.

The hazard ratios (HR) of progression and death, the odds ratios (OR) of response and their associated 95\% confidence intervals (95\% CI) for clinically important factors (including PS, histology, stage, gender, age, line of treatment, TTP to previous line of chemotherapy, PD-L1 status) were calculated using a univariable Cox proportional hazard model or a univariable logistic regression.

Missing data in blood-derived parameters analyzed in the multivariable analysis were imputed ten times. In order to determine a subset of variables with the strongest impact on PFS, OS and ORR, blood-derived biomarkers (including albumin, platelets, absolute neutrophils, monocytes, eosinophils and lymphocytes) were combined with clinically important factors and a Cox multivariable proportional hazard regression model or a multivariable logistic regression were performed on the imputed datasets. Since the number of candidate variables exceeded the number of events divided by 10 , a ridge version of the models was used for variable selection. Variables were selected in the final model if they were included 5 times of more in the models on the imputed data sets. The final model was fitted on the imputed data sets and the results were pooled using Rubin's rules (19). As a sensitivity analysis, the final model was also estimated on the complete case data (without imputed data).

Associations between categorical variables were assessed by Pearson's Chi-Square or Fisher exact tests.

A significance level of 0.05 was chosen to assess the statistical significance. All reported $\mathrm{P}$ values were two sided. Statistical analyses were performed using R 3.6.0 (R Foundation for Statistical Computing). Multiple imputation was performed using the "smcfcs" package and pooling was conducted with the "mice" and "mitools" packages in $\mathrm{R}$.

As data in our cohort were collected retrospectively, no approval by a medical research and ethics committee was necessary according to Dutch guidelines (https://english. ccmo.nl).

\section{Results}

\section{Patient characteristics}

At the data cut-off of November 2019, 135 patients were 
Table 1 Patient baseline characteristics

\begin{tabular}{|c|c|}
\hline Characteristic & Outcome (total=107) \\
\hline Median age [range] (years) & 69 [34-84] \\
\hline Gender, male, n [\%] & $95[87]$ \\
\hline \multicolumn{2}{|c|}{ ECOG PS at start of nivolumab, $n$ [\%] } \\
\hline 0 & 20 [19] \\
\hline 1 & 68 [64] \\
\hline 2 & $6[5]$ \\
\hline Unknown & $13[12]$ \\
\hline \multicolumn{2}{|l|}{ Histological subtype, n [\%] } \\
\hline Epithelioid & 78 [73] \\
\hline Mixed/sarcomatoid & $22[20]$ \\
\hline Unknown & 7 [7] \\
\hline \multicolumn{2}{|c|}{ Best response to previous platinum-based chemotherapy, $\mathrm{n}$ [\%] } \\
\hline PD & $28[26]$ \\
\hline SD & $46[43]$ \\
\hline PR & $28[26]$ \\
\hline $\mathrm{CR}$ & $1[1]$ \\
\hline Unknown (not reported) & $4[4]$ \\
\hline \multicolumn{2}{|l|}{ Line of treatment, $\mathrm{n}$ [\%] } \\
\hline 2 & 97 [91] \\
\hline$\geq 3$ & $10[9]$ \\
\hline \multicolumn{2}{|c|}{ Stage at start of nivolumab, $\mathrm{n}[\%]$} \\
\hline $\mathrm{I} / \mathrm{II}$ & $32[30]$ \\
\hline III/IV & 70 [65] \\
\hline Unknown & $5[5]$ \\
\hline \multicolumn{2}{|l|}{ PD-L1 status, $n$ [\%] } \\
\hline Negative & $22[20]$ \\
\hline Positive & $11[10]$ \\
\hline Unknown & 74 [70] \\
\hline
\end{tabular}

Data are presented as absolute number with according percentages, unless stated otherwise. ECOG PS, Eastern Cooperative Oncology Group Performance Status; PD, progressive disease; SD, stable disease; $\mathrm{PR}$, partial response; $\mathrm{CR}$, complete response; PD-L1, programmed death ligand 1.

treated with at least one cycle of Nivolumab. Among them, 107 patients were eligible for the analysis (Figure S1). Eighty-eight patients (93\%) had a PS of 0 or 1 at start of treatment. Ninety-seven (90\%) were treated in second- line. PD-L1 expression was determined in 33 patients: 22 biopsies (66\%) were PD-L1 negative and 11 (33\%) were PD-L1 positive. PD-L1 positive status was associated with non-epithelioid histology (Fisher's exact test $\mathrm{P}$ value 0.004 ). The majority of patients (69\%) had an advanced clinical stage of disease (stage III/IV). Other baseline patient characteristics are summarized in Table 1.

At a median follow-up time of 10.1 months, 85 patients had progression of disease of whom 59 died. The median PFS (mPFS) was 2.3 months (95\% CI: 1.6-2.9) and median OS (mOS) was 6.7 months (95\% CI: 6.2-10.0) (Figure $1 A, B)$. The disease control rate (DCR) was $37 \%$ (40 out of 107) after 12 weeks and 11 patients (10\%) had an objective radiological response (all partial responders, no complete responses were registered). The 6-month PFS rate was $23 \%$ (95\% CI: $16-33 \%)$. The 6-month OS rate was 60\% (95\% CI: $51-71 \%)$ and the 1 -year OS rate was $31 \%$ (95\% CI: $22-45 \%)$.

\section{Association of clinically important factors with survival outcomes}

Univariable Cox proportional hazard regression analysis of clinically important factors revealed that patients with advanced clinical stage (stage III/IV) had a shorter PFS [mPFS 1.6 vs. 3.6 months (HR 1.82, 95\% CI: 1.11-3.01, log-rank $\mathrm{P}$ value 0.02 , Figure $2 A$ )] but similar OS [mOS 6.5 vs. 6.8 months (HR 1.27, 95\% CI: 0.71-2.28, log-rank P value 0.40 ), Figure $2 B$ ] compared to those with early stage (I/II). All other clinical factors were not significantly associated with PFS or OS (Table 2).

In particular, PS was not significantly correlated with PFS or OS, although patients with a PS of 0 had a trend towards a longer mOS compared to patients with PS $\geq 1$ [mPFS 2.9 vs. 1.8 months (HR 0.64, 95\% CI: 0.36-1.16, log-rank $\mathrm{P}$ value 0.14 ); mOS 10.2 vs. 6.2 months (HR 0.51, 95\% CI: $0.25-1.05$, log-rank P value 0.06 )]. PFS was also similar among patients with non-epithelioid and epithelioid histology (log-rank $\mathrm{P}$ value 0.89 , Figure $2 C$ ), yet patients with non-epithelioid histology had a non-significant trend towards worse OS [mOS 4.8 vs. 7.4 months (HR 1.71, 95\% CI: $0.92-3.16$, log-rank P value 0.08), Figure 2D]. Patients with positive PD-L1 status showed a longer, albeit non-significant, mPFS [4.2 vs. 1.7 months (HR 0.52, 95\% CI: $0.23-1.20, \log$-rank $\mathrm{P}$ value 0.11 ), Figure $2 E]$ while no difference in terms of OS was observed [mOS $5.4 \mathrm{vs}$. 6.1 months (HR 0.67, 95\% CI: 0.27-1.64, log-rank P value $0.39)$, Figure $2 F]$. 

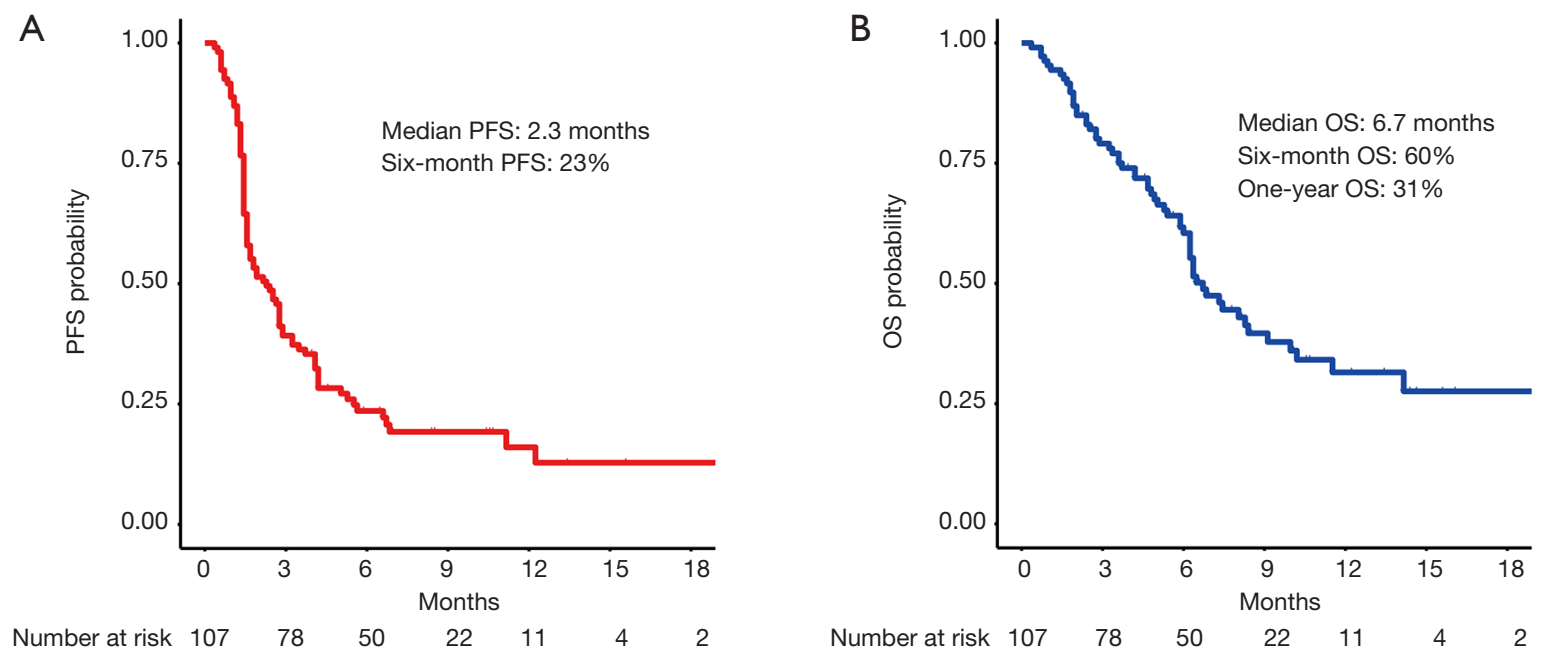

Figure 1 Kaplan-Meier curves of survival in the entire cohort of nivolumab treated MPM patients (median follow-up time of 10.1 months). (A) Progression-free survival in the entire cohort; (B) overall survival in the entire cohort. PFS, progression-free survival; OS, overall survival.

\section{Impact of radiological response to nivolumab on outcome and association of clinically important factors with response}

To better elucidate the importance of response to nivolumab, we compared PFS and OS of patients according to ORR. To avoid an immortal time bias, only patients who were still alive at 12 weeks and underwent radiological assessment at that time point were taken into account for the analysis. Remarkably, with a median follow up of 14.1 months in the group of patients with PR, no deaths were reported and only 2 patients progressed (median DoR not reached, Figure $3 A$ ). Median OS was not reached for patients with a PR. Median OS was 10.2 months for patients with SD and 6.4 months for those with PD (logrank P value 0.0002 , Figure $3 B$ ). Among the clinically relevant factors, the only one which seemed to predict ORR in univariable logistic regression was PD-L1 status (Table 2). To note, data about PD-L1 expression were only available in 6/11 PR, 8/29 SD and 19/67 PD patients (Figure 4). Four of the responders had PD-L1 positive tumors and two had PD-L1 negative tumors (Figure 4). ORR was $36 \%$ in the PD-L1 positive group vs. $9 \%$ in the PD-L1 negative group (OR 1.31, 95\% CI: 1.00-1.72, P value 0.05, Table 2).

\section{Association of peripheral blood biomarkers with survival outcomes and response to nivolumab}

After imputation for missing values (refer to Figure S1 for the number of available blood samples at baseline), peripheral blood-derived parameters (albumin, platelets, absolute neutrophils, monocytes, eosinophils and lymphocytes) and clinically important factors (including PS, histology, clinical stage, gender and age) were used as covariates in multivariable analysis to identify independent factors related to the efficacy of nivolumab in terms of PFS and OS. Regarding PFS, only high absolute monocyte count was significantly associated with worse PFS after ridge regression (HR 3.16, 95\% CI: 1.56-6.37, $\mathrm{P}$ value 0.001 , Table 3). The role of monocytes was confirmed also by using non-imputed data (HR 3.78, 95\% CI: 1.84-7.76, P value 0.0002 ).

The ridge regression for OS showed that albumin, thrombocytes, neutrophils had the strongest association with OS. Subsequent multivariable Cox proportional hazard regression analysis with these variables (Table 3) showed that only albumin retained its prognostic value revealing that patients with a high albumin had a lower change of dying (HR 0.87, 95\% CI: 0.81-0.95, P value 0.002). The role of albumin was confirmed by the sensitivity analysis with nonimputed data (HR 0.88, 95\% CI: $0.80-0.96$, P value 0.005 ).

A multivariable analysis for ORR with peripheral bloodderived parameters was not performed because of the low number of events (only 11 responder patients). At univariable analysis with imputed data, again only albumin resulted significantly associated with ORR (OR 1.02, 95\% CI: $1.00-1.03, \mathrm{P}$ value 0.03 , Table S1).

Since albumin was the only significant prognostic factor 

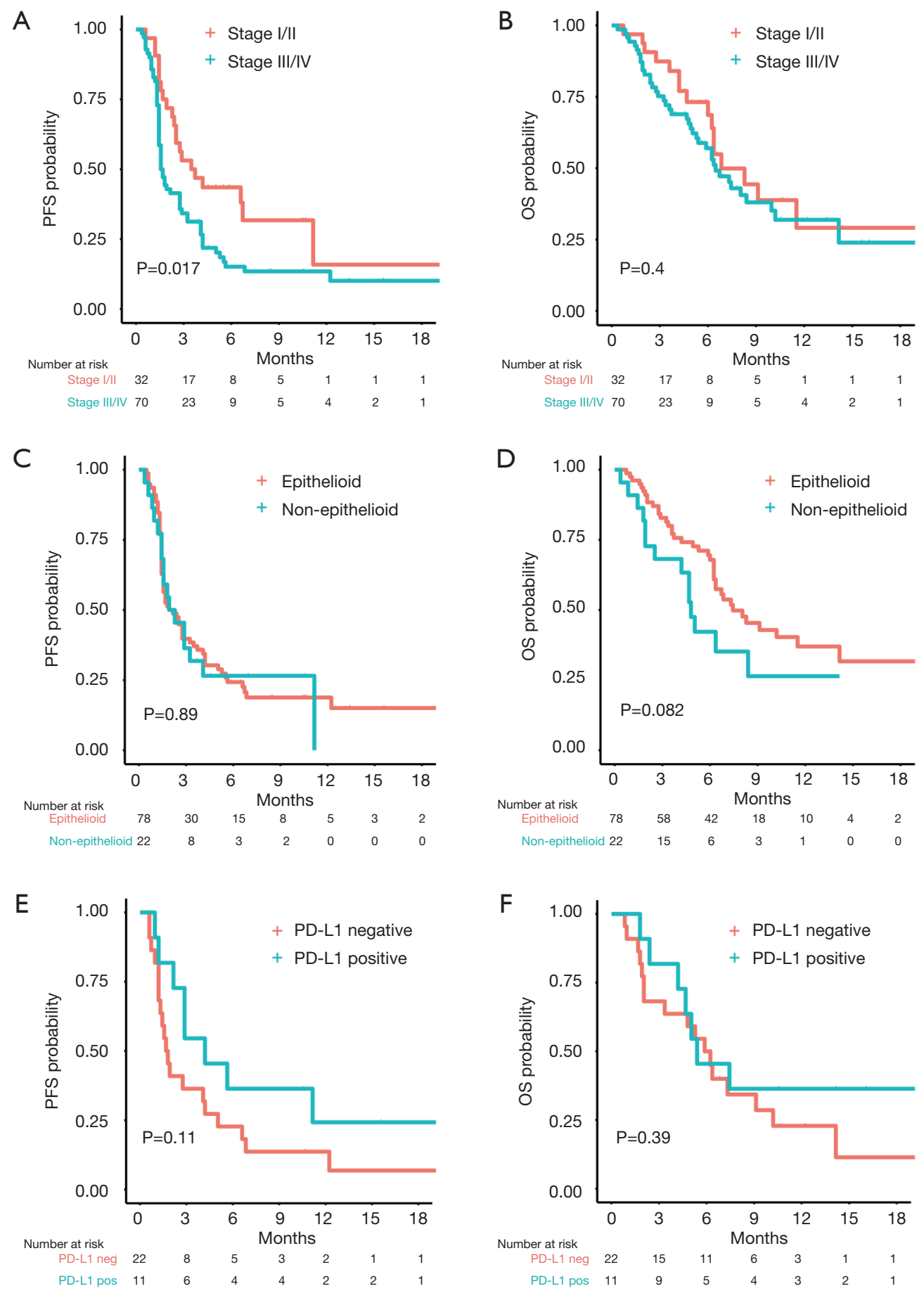

Figure 2 Kaplan-Meier curves of survival of subgroups based on stage of disease, histological subtype and programmed death ligand 1 (PDL1) status. (A) Progression-free survival and (B) overall survival by stage of disease as determined by IASLC $8^{\text {th }}$ edition of TNM for pleural mesothelioma. (C) Progression-free survival and (D) overall survival by histology. (E) Progression-free survival and (F) overall survival in patients with a PD-L1 expression $\geq 1 \%$ versus in those with a PD-L1 expression $<1 \%$. PFS, progression-free survival; OS, overall survival; PD-L1, programmed death ligand 1. 
Table 2 Univariable analysis of PFS, OS and ORR for clinically important factors

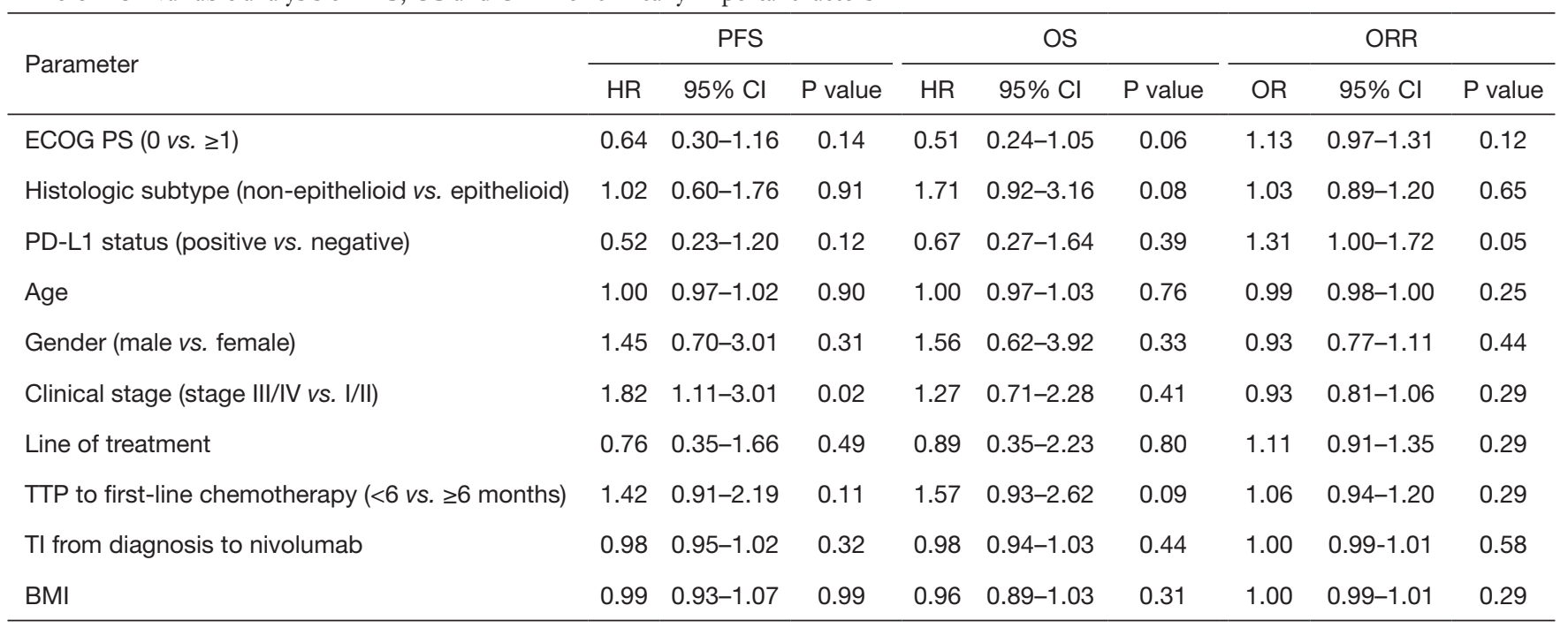

The univariable Cox proportional hazard model was used to calculate the HRs of progression or death and the univariable logistic regression was used to calculate the ORs of response. PFS, progression-free survival; OS, overall survival; ORR, objective response rate; HR, hazard ratio; OR, odds ratio; Cl, confidence interval; ECOG PS, Eastern Cooperative Oncology Group Performance Status; PD-L1, programmed death ligand 1; TTP, time to progression; TI, time interval; BMI, body mass index.

A

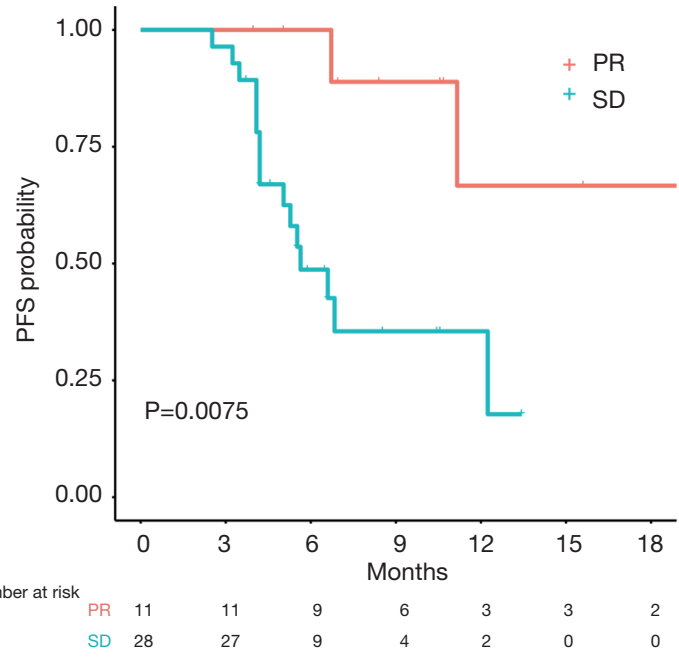

B

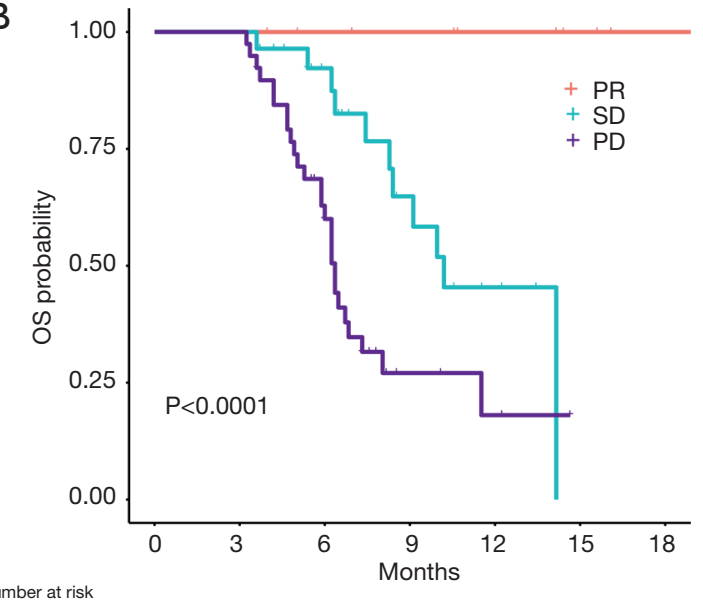

Number at risk

$\begin{array}{cccccccc}\text { PR } & 11 & 11 & 9 & 8 & 6 & 4 & 2 \\ \text { SD } & 28 & 28 & 19 & 10 & 3 & 0 & 0 \\ \text { PD } & 39 & 39 & 22 & 4 & 2 & 0 & 0\end{array}$

Figure 3 Kaplan-Meier curves of survival according to best overall radiological response. (A) Progression-free survival in patients with a partial response and stable disease as objective response to nivolumab treatment; (B) overall survival in patients with a partial response, stable disease and progressive disease as objective response to nivolumab treatment. PFS, progression-free survival; OS, overall survival; PR, partial response; $\mathrm{SD}$, stable disease; $\mathrm{PD}$, progressive disease. 
for OS and was also associated with ORR in univariable analysis, patients were further divided in quartiles according to their baseline albumin values and their outcomes were analyzed. Patients in the lower quartile $(<38 \mathrm{mg} / \mathrm{dL})$ revealed a significantly shorter OS compared to patients in the other quartiles (HR 3.76, 95\% CI: 1.93-7.31, log-rank $\mathrm{P}$ value 0.003 with Bonferroni's correction, Figure 5). The median OS for patient with baseline albumin levels below 38 was 2.5 months (95\% CI: 1.9 -not reached) compared to 8.0 months (95\% CI: $6.4-$ not reached) for patients with albumin levels above 38 . Six-month OS rates were $34 \%$ (95\% CI: $18-65 \%$ ) and $74 \%$ (95\% CI: $62-86 \%$ ), respectively. In addition, 4 out of $20(20 \%)$ patients in the higher quartile $(>43 \mathrm{mg} / \mathrm{dL})$ had a partial response,

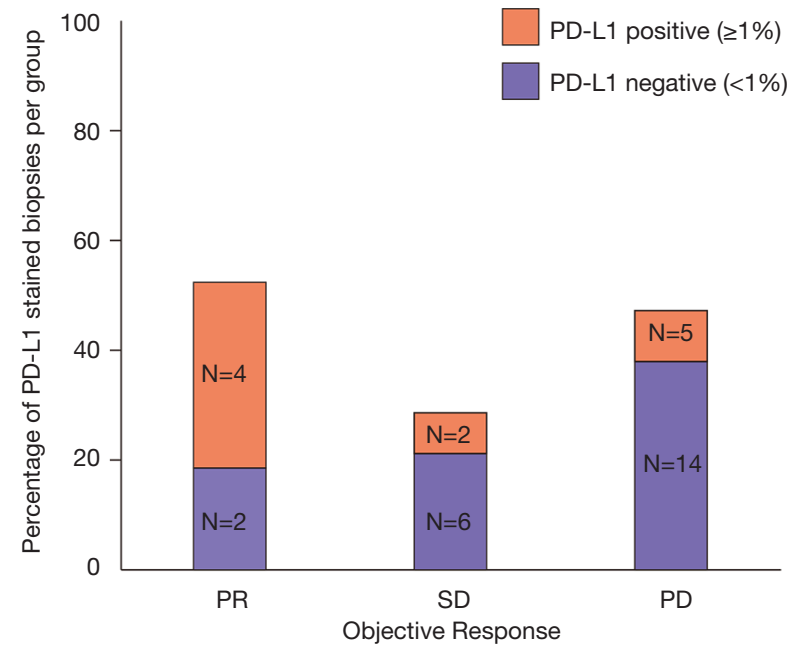

Figure 4 Expression of programmed death ligand 1 (PD-L1) according to objective response to nivolumab treatment. PR, partial response; $\mathrm{SD}$, stable disease; $\mathrm{PD}$, progressive disease; $\mathrm{PD}$ L1, programmed death ligand 1. compared to $3 / 65(4 \%)$ in the other three quartiles, with a $16 \%$ increase in the chance of getting a response to nivolumab (OR 1.16, 95\% CI: 1.02-1.33, P value 0.02).

\section{Discussion}

This is the largest real-world analysis of nivolumab treatment in pre-treated MPM patients. We observed an ORR of $10 \%$, a mPFS of 2.3 months and a mOS of 6.7 months. The PFS and OS did not significantly differ per histological subtype or PD-L1 expression. Patients

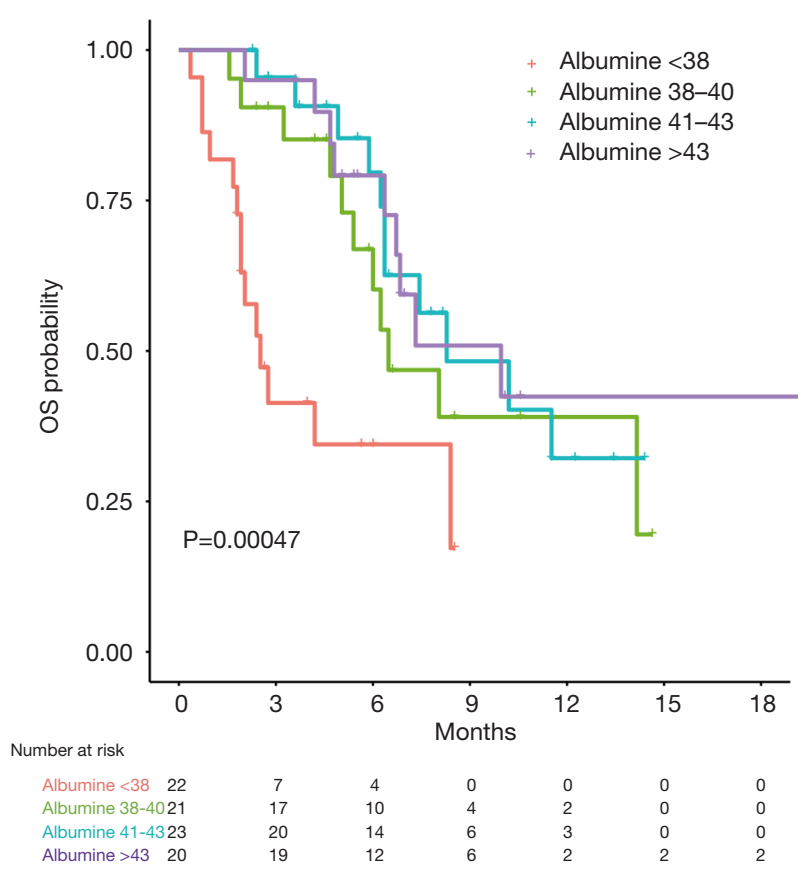

Figure 5 Kaplan-Meier curves of survival in patient groups per quartile of albumin level. OS, overall survival.

Table 3 Multivariable analysis of PFS and OS for peripheral blood derived parameters

\begin{tabular}{|c|c|c|c|c|c|c|}
\hline Parameter & \multicolumn{3}{|c|}{ PFS } & \multicolumn{3}{|c|}{ os } \\
\hline Monocytes $(/ \mu \mathrm{L})$ & 3.16 & $1.56-6.37$ & 0.001 & & & \\
\hline Albumin (mg/dL) & & & & 0.87 & $0.81-0.95$ & 0.002 \\
\hline Platelet count $(/ \mu \mathrm{L})$ & & & & 1.00 & $0.99-1.01$ & 0.07 \\
\hline
\end{tabular}

Only variables that came out more than five times from the ridge regression in the imputed data set were included in this final model. The final model was fitted on the imputed data sets and the results were pooled using Rubin's rules. Co-variables for ridge regression included PS, histology, stage, gender, age, eosinophils and lymphocytes. PFS, progression-free survival; OS, overall survival, HR, hazard ratio; Cl, confidence interval. 
with PD-L1 positive tumors had a higher ORR than patients with PD-L1 negative tumors. We did not observe an association between time from diagnosis or response to chemotherapy and response to nivolumab. Strikingly, there seemed to be an incremental impact on OS for patients with a PR to nivolumab as we did not observe any deaths in these patients during a median follow-up time of 14.1 months.

By comparing our data with the real-world study of MPM patients treated with pembrolizumab, we observed a similar OS but a worse PFS, which could be explained by the type of radiological assessment used. In the study of Metaxas et al. (12), the type of radiological assessment was not described. In our study, we retrospectively analyzed all CT scans according to a combination of mRECIST for mesothelioma and iRECIST $(16,17)$. Per iRECIST, tumor assessment had to be repeated 4 to 8 weeks after first evidence of PD with the option of continuing treatment if the patient was clinically stable. In case of confirmed progression, the date of the initial CT scan was taken as the time of progression.

By comparing our data with those of clinical trials (7-11), our ORR and mOS were inferior, which could be explained by the fact that there were no strict inclusion criteria in our analysis, leading to a less selected patient population. In the PROMISE-meso trial an ORR of $22 \%$ was reported for the pembrolizumab group and an ORR of $6 \%$ for the second-line chemotherapy treated patients. However, this difference in ORR was not translated into a difference in mPFS (pembrolizumab: 2.5 months $v s$. chemotherapy: 3.4 months) or mOS (pembrolizumab: 10.7 months $v s$. chemotherapy: 11.7 months) (7). Conversely, long survival for patients with a PR in our analysis does suggest a clinical benefit that is correlated with ORR. The lack of significant benefit in terms of mPFS and mOS, despite a higher ORR, in the pembrolizumab arm of the PROMISE-meso might be due to the low ORR combined with the short time to progression in patients where therapy is not effective. For example, if only a minority of patients (10-20\%) respond to therapy, mPFS and mOS will not be influenced, because more than $50 \%$ of the patients will progress or die earlier according to the natural course of disease. Six-months PFS and 1-year OS might be more reliable endpoints for (immune) therapies with low response rates. Analysis of those patients who achieved a PR to pembrolizumab in the PROMISE-meso study has not yet been published but could be explanatory.

Since retrospective data may be biased by underreporting of adverse events and misleading, we decided not to report safety data. Nevertheless, to avoid a potentially harmful treatment, identifying a subgroup of MPM patients that benefit from nivolumab becomes crucial. This patient selection should probably be based on multiple parameters.

MPM patients with epithelioid histology have usually a better natural disease course than patients with nonepithelioid tumors (20). However, in our retrospective analysis we did not see any significant difference in mPFS and mOS according to histological subtypes, suggesting that nivolumab might have had an impact on prognosis of non-epithelioid patients. Moreover, PD-L1 expression was associated with non-epithelioid histology and higher ORR in our study. These results are consistent with the exploratory analysis of the MAPS2 trial, where PDL1 expression of $\geq 1 \%$ was found to be significantly associated with objective response to immunotherapy (8). Unfortunately, our analysis on PD-L1 expression was limited because only $30 \%$ of biopsies were stained for PD-L1. Another limitation is that PD-L1 expression was often determined on the biopsy from diagnosis, because in most cases there was no biopsy taken prior to nivolumab treatment.

Looking at the role of baseline peripheral blood biomarkers, our study showed that baseline albumin was the only significant prognostic factor for mOS. In addition, patients with an albumin level higher than $43 \mathrm{mg} / \mathrm{dL}$ had a $16 \%$ higher chance of responding to therapy than patients with albumin levels below $38 \mathrm{mg} / \mathrm{dL}$. Albumine is known to reflect the nutritional status of cachectic patients and is described as a prognostic factor for many cancer types, including mesothelioma (21-23). Due to the lack of a control group, we cannot draw definitive conclusions about the predictive role of albumin from our analysis. However, we showed that low levels of albumin might identify patients who are unlikely to benefit from the treatment.

Our analysis also showed that baseline absolute monocyte count represents an optimal predictor of PFS in MPM patients (HR 3.16, 95\% CI: 1.56-6.37, P value 0.001 ). This negative association between the number of monocytes and outcome in MPM is consistent with previous studies $(24,25)$. Burt et al. reported that pre-operative peripheral absolute monocyte count was associated with poor OS in patients with MPM, regardless of tumor histology (HR 3.98, 95\% CI: 2.64-5.93, $\mathrm{P}$ value $<0.0001)(25)$.

\section{Conclusions}

In conclusion, our study showed that ORR and mOS 
were lower in our real-world database compared to those of clinical trials, which could be due to a less selected population. However, we identified a subgroup of MPM patients with a radiological response to nivolumab that had a significant benefit in terms of PFS and OS compared to patients without a radiological response to nivolumab treatment. We also showed that PD-L1 expression and albumin were associated with higher response rate, yet the retrospective nature of our study and the lack of a control group prevent us from drawing definitive conclusions on their role as potential predictive biomarkers. Future phase III RCTs on CI treatment in MPM should not be conducted without an extensive exploratory analysis plan based on the evaluation of peripheral blood parameters and tumor samples in order to deeply characterize the small group of patients that benefit from CI treatment.

\section{Acknowledgments}

We thank Peter van Gelder for language editing assistance. Funding: None.

\section{Footnote}

Reporting Checklist: The authors have completed the STROBE Reporting Checklist. Available at http://dx.doi. org/10.21037/tlcr-19-686

Data Sharing Statement: Available at http://dx.doi. org/10.21037/tlcr-19-686

Conflicts of Interest: All authors have completed the ICMJE uniform disclosure form (available at http://dx.doi. org/10.21037/tlcr-19-686). DWD reports personal fees from Roche, BMS, MSD, Pfizer, Astra Zeneca and Novartis outside the submitted work. RC reports speakers fee from Roche, Pfizer and BMS, personal fees from Advisory Board MSD and Advisory Board Roche outside the submitted work. JAB reports other from Bristol-Meyers Squibb, MSD B.V., F. Hoffmann- La Roche Ltd. during the conduct of the study. PB reports other from Bristol-Myers Squibb, MSD B.V., AstraZeneca, Takeda outside the submitted work. JGJVA reports personal fees and non-financial support from MSD, personal fees from BMS, Boehringer Ingelheim, Amphera, Eli-Lilly, Takeda, Bayer, Roche, Astra Zeneca outside the submitted work; in addition, JGJVA has a patent allogenic tumor cell lysate licensed to Amphera, a patent combination immunotherapy in cancer pending, and a patent biomarker for immunotherapy pending; JGJVA serves as an unpaid editorial board member of Translational Lung Cancer Research from Sep 2019 to Sep 2021. The other authors have no conflicts of interest to declare. The authors declare that the research was conducted in the absence of any commercial or financial relationships that could be construed as a potential conflict of interest.

Ethical Statement: The authors are accountable for all aspects of the work in ensuring that questions related to the accuracy or integrity of any part of the work are appropriately investigated and resolved. As data in our cohort were collected retrospectively, no approval by a medical research and ethics committee was necessary according to Dutch guidelines (https://english.ccmo.nl).

Open Access Statement: This is an Open Access article distributed in accordance with the Creative Commons Attribution-NonCommercial-NoDerivs 4.0 International License (CC BY-NC-ND 4.0), which permits the noncommercial replication and distribution of the article with the strict proviso that no changes or edits are made and the original work is properly cited (including links to both the formal publication through the relevant DOI and the license). See: https://creativecommons.org/licenses/by-nc-nd/4.0/.

\section{References}

1. Vogelzang NJ, Rusthoven JJ, Symanowski J, et al. Phase III study of pemetrexed in combination with cisplatin versus cisplatin alone in patients with malignant pleural mesothelioma. J Clin Oncol 2003;21:2636-44.

2. Zalcman G, Mazieres J, Margery J, et al. Bevacizumab for newly diagnosed pleural mesothelioma in the Mesothelioma Avastin Cisplatin Pemetrexed Study (MAPS): A randomised, controlled, open-label, phase 3 trial. Lancet 2016;387:1405-14.

3. van Meerbeeck JP, Gaafar R, Manegold C, et al. Randomized phase III study of cisplatin with or without raltitrexed in patients with malignant pleural mesothelioma: An intergroup study of the European organisation for research and treatment of cancer lung cancer group and the National Cancer Institute. J Clin Oncol 2005;23:6881-9.

4. Mansfield AS, Zauderer MG. Nivo-lution in mesothelioma. Clin Cancer Res 2019;25:5438-40.

5. Cantini L, Hassan R, Sterman DH, et al. Emerging Treatments for Malignant Pleural Mesothelioma: Where 
Are We Heading? Front Oncol 2020;10:343.

6. Maio M, Scherpereel A, Calabrò L, et al. Tremelimumab as second-line or third-line treatment in relapsed malignant mesothelioma (DETERMINE): a multicentre, international, randomised, double-blind, placebocontrolled phase 2b trial. Lancet Oncol 2017;18:1261-73.

7. Popat S, Curioni-Fontecedro A, Polydoropoulou V, et al. LBA91_PRA multicentre randomized phase III trial comparing pembrolizumab $(\mathrm{P})$ vs single agent chemotherapy (CT) for advanced pre-treated malignant pleural mesothelioma (MPM): Results from the European Thoracic Oncology Platform (ETOP 9-15) PROMISEmeso tri. Ann Oncol 2019;30:mdz394.091.

8. Scherpereel A, Mazieres J, Greillier L, et al. Nivolumab or nivolumab plus ipilimumab in patients with relapsed malignant pleural mesothelioma (IFCT-1501 MAPS2): a multicentre, open-label, randomised, non-comparative, phase 2 trial. Lancet Oncol 2019;20:239-53.

9. Quispel-Janssen J, van der Noort V, de Vries JF, et al. Programmed Death 1 Blockade With Nivolumab in Patients With Recurrent Malignant Pleural Mesothelioma. J Thorac Oncol 2018;13:1569-76.

10. Disselhorst MJ, Quispel-Janssen J, Lalezari F, et al. Ipilimumab and nivolumab in the treatment of recurrent malignant pleural mesothelioma (INITIATE): results of a prospective, single-arm, phase 2 trial. Lancet Respir Med 2019;7:260-70.

11. Okada M, Kijima T, Aoe K, et al. Clinical efficacy and safety of nivolumab: results of a multicenter, open-label, single-arm, Japanese phase 2 study in malignant pleural mesothelioma (MERIT). Clin Cancer Res 2019;25:5485-92.

12. Metaxas Y, Rivalland G, Mauti LA, et al. Pembrolizumab as Palliative Immunotherapy in Malignant Pleural Mesothelioma. J Thorac Oncol 2018;13:1784-91.

13. Tanizaki J, Haratani K, Hayashi H, et al. Peripheral Blood Biomarkers Associated with Clinical Outcome in NonSmall Cell Lung Cancer Patients Treated with Nivolumab. J Thorac Oncol 2018;13:97-105.

14. Weide B, Martens A, Hassel JC, et al. Baseline biomarkers for outcome of melanoma patients treated with pembrolizumab. Clin Cancer Res 2016;22:5487-96.

15. Brierley JD, Gospodarowicz MK, Wittekind C. TNM classification of malignant tumours. 8th edition. Union for International Cancer Control. 2016.

16. Tsao AS, Garland L, Redman M, et al. A practical guide of the southwest oncology group to measure malignant pleural mesothelioma tumors by RECIST and modified RECIST criteria. J Thorac Oncol 2011;6:598-601.

17. Seymour L, Bogaerts J, Perrone A, et al. iRECIST: guidelines for response criteria for use in trials testing immunotherapeutics. Lancet Oncol 2017;18:e143-52.

18. Anderson JR, Cain KC, Gelber RD. Analysis of survival by tumor response. J Clin Oncol 1983;1:710-9.

19. Barnard J, Rubin DB. Small-sample degrees of freedom with multiple imputation. Biometrika 1999;86:948-55.

20. Van Schil PE, Opitz I, Weder W, et al. Multimodal management of malignant pleural mesothelioma: Where are we today? Eur Respir J 2014;44:754-64.

21. Yang JR, Xu JY, Chen GC, et al. Post-diagnostic C-reactive protein and albumin predict survival in Chinese patients with non-small cell lung cancer: a prospective cohort study. Sci Rep 2019;9:8143.

22. Ikeda S, Yoshioka H, Ikeo S, et al. Serum albumin level as a potential marker for deciding chemotherapy or best supportive care in elderly, advanced non-small cell lung cancer patients with poor performance status. BMC Cancer 2017;17:797.

23. Yao ZH, Tian GY, Yang SX, et al. Serum albumin as a significant prognostic factor in patients with malignant pleural mesothelioma. Tumour Biol 2014;35:6839-45.

24. Yamagishi T, Fujimoto N, Nishi H, et al. Prognostic significance of the lymphocyte-to-monocyte ratio in patients with malignant pleural mesothelioma. Lung Cancer 2015;90:111-7.

25. Burt BM, Rodig SJ, Tilleman TR, et al. Circulating and tumor-infiltrating myeloid cells predict survival in human pleural mesothelioma. Cancer 2011;117:5234-44.
Cite this article as: Cantini L, Belderbos RA, Gooijer CJ, Dumoulin DW, Cornelissen R, Baart S, Burgers JA, Baas $\mathrm{P}$, Aerts JGJV. Nivolumab in pre-treated malignant pleural mesothelioma: real-world data from the Dutch expanded access program. Transl Lung Cancer Res 2020;9(4):1169-1179. doi: 10.21037/tlcr-19-686 


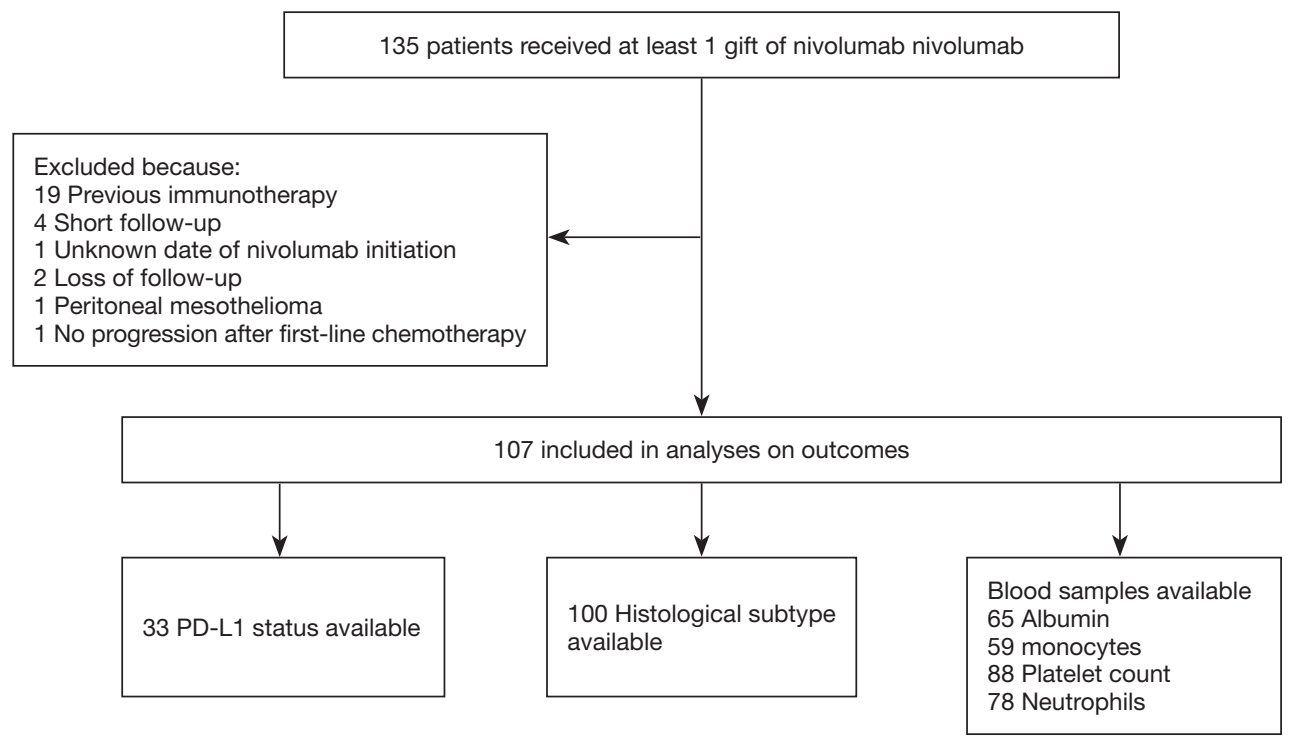

Figure S1 Flow diagram of study population. PD-L1, programmed death ligand 1.

Table S1 Univariable analysis of ORR for peripheral blood derived parameters

\begin{tabular}{lccc}
\hline \multirow{2}{*}{ Parameter } & & ORR & P value \\
\cline { 2 - 4 } & OR & $95 \% \mathrm{Cl}$ & 0.03 \\
\hline Albumin $(\mathrm{mg} / \mathrm{dL})$ & 1.02 & $1.00-1.03$ & 0.42 \\
Platelet count $(/ \mu \mathrm{L})$ & 0.99 & $0.99-1.00$ & 0.28 \\
Neutrophils $(/ \mu \mathrm{L})$ & 0.98 & $0.96-1.00$ & 0.99 \\
Lymphocytes $(/ \mu \mathrm{L})$ & 1.00 & $0.93-1.06$ & 0.89 \\
Monocytes $(/ \mu \mathrm{L})$ & 0.98 & $0.78-1.22$ & 0.30 \\
Eosinophils $(/ \mu \mathrm{L})$ & 0.75 & $0.44-1.28$ & \\
\hline
\end{tabular}

The univariable logistic regression was used to calculate the ORs of response for peripheral blood derived parameters (with imputed data). ORR, objective response rate; OR, odds ratio; $\mathrm{Cl}$, confidence interval. 